\title{
Fifth DOE Information Meeting on High Tc Superconductors Explores Effects of Microstructure
}

\section{Terry L. Aselage and Keith D. Keefer}

The theme of the Fifth U.S. Department of Energy Information Meeting on High Temperature Superconductivity was the "Effect of Microstructure on the Properties of High Temperature Superconducting Materials." Sponsored by the Materials Science Division of the Office of Basic Energy Sciences of DOE, the meeting was held at Sandia National Laboratories, Albuquerque, New Mexico, January 25 and 26, 1988. Approximately 150 scientists from around the United States attended.

The purpose of these meetings has been to exchange information on the new high temperature superconductors in a timely fashion and to coordinate superconductivity research among the various DOE-sponsored programs. To broaden participation, this meeting featured a live, nationwide television broadcast of the program by satellite hookup through the National Technical University.

Special requirements dictated by the broadcast necessitated a different format than that used in previous meetings, according to Jim Schirber of Sandia National Laboratories, who organized the meeting. In order to keep to the tight schedule yet allow for free discussion, a panel format was utilized. Five panels were assigned the task of discussing the following topics: (1) interconnects and critical currents, (2) structural properties and twins, (3) oxygen stoichiometry, vacancies and doping, (4) pinning, magnetic anisotropy and critical fields, and (5) theory. Each panel member had five minutes to describe an aspect of the topic, after which the

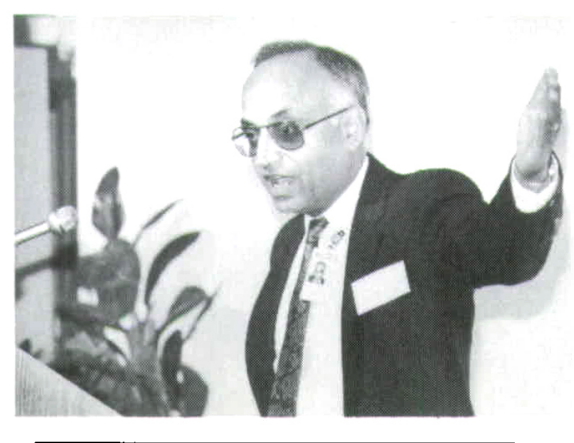

V. Narayanamurti, vice president of research at Sandia, welcomes participants to the DOE information meeting on the "Effect of Microstructure of the Properties of High Temperature Superconductors." (Photo, R. Montoya, Sandia)

panel members answered questions from other panelists, the studio audience, and television viewers who telephoned their questions. A late afternoon session, which was not televised, allowed for the presentation of recent research results.

\section{Panel 1-Interconnects and Critical Currents}

The first panel on interconnects and critical currents focused on intergranular weak links in polycrystalline ceramic materials. A number of effects at or near grain boundaries were identified, including second phases (primarily $\mathrm{BaCuO}_{2}$ and $\mathrm{CuO}$ ), cracks resulting from thermal expansion anisotropy, carbonate impurities, nonstoichiometry, and anisotropy in transport properties (grain alignment). G. Fisanick (AT\&T Bell Laboratories) reported scanning Auger results suggesting that a $10 \AA$ insulating carbonate layer is present at grain boundaries. R. McCallum (Ames Laboratory) followed by presenting results that showed that much of the carbonate found at grain boundaries in their samples results from exposure to laboratory air for long periods of time. Cracks at grain boundaries were cited as an important factor. D. Kroeger (Oak Ridge National Laboratory) also observed no carbon in fracture surfaces, and suggested that stoichiometry differences at grain boundaries are important. D. Capone (Argonne National Laboratory) pointed out that texturing of their samples did not lead to significant increases in $\mathrm{J}_{c}$, indicating that grain orientation alone was not enough to solve the weak link problem. Several panelists pointed out that the presence of such grain boundary effects were linked to processing history, and that each of the effects could limit the currents. The consensus was that the grain boundaries need to be cleaned up first, followed by orientation of the grains. The recent results of $S$. Jin and coworkers (AT\&T Bell Laboratories) on melt textured samples were cited as evidence of the possibility of obtaining high critical currents in polycrystalline samples.

\section{Panel 2-Structural Properties and Twins}

The second panel on structural properties and twins focused primarily on twins. R. Gronsky (Lawrence Berkeley Laboratory) presented evidence of a rotational twin present in quenched samples of tetragonal 1-2-3. These rotational twins may inhibit the orthorhombic to tetragonal transition. S. Babcock (University of Wisconsin) used STEM to study grain boundaries, concluding that the critical problems may be related to compositional gradients near boundaries. T. Mitchell (Los Alamos National Laboratory) presented work showing that twins can be formed by thermal stresses in TEM foils, while M. Suenaga (Brookhaven National Laboratory) showed that fine twins are generated by doping with Fe. Damage of samples due to milling and beam radiation was discussed; the observation was made that samples should be examined quickly to avoid such damage. B. Morosin (Sandia National Laboratories) discussed the effect of high pressure oxygen loading on the crystal structure of $\mathrm{La}_{2} \mathrm{CuO}_{4}$, suggesting that 


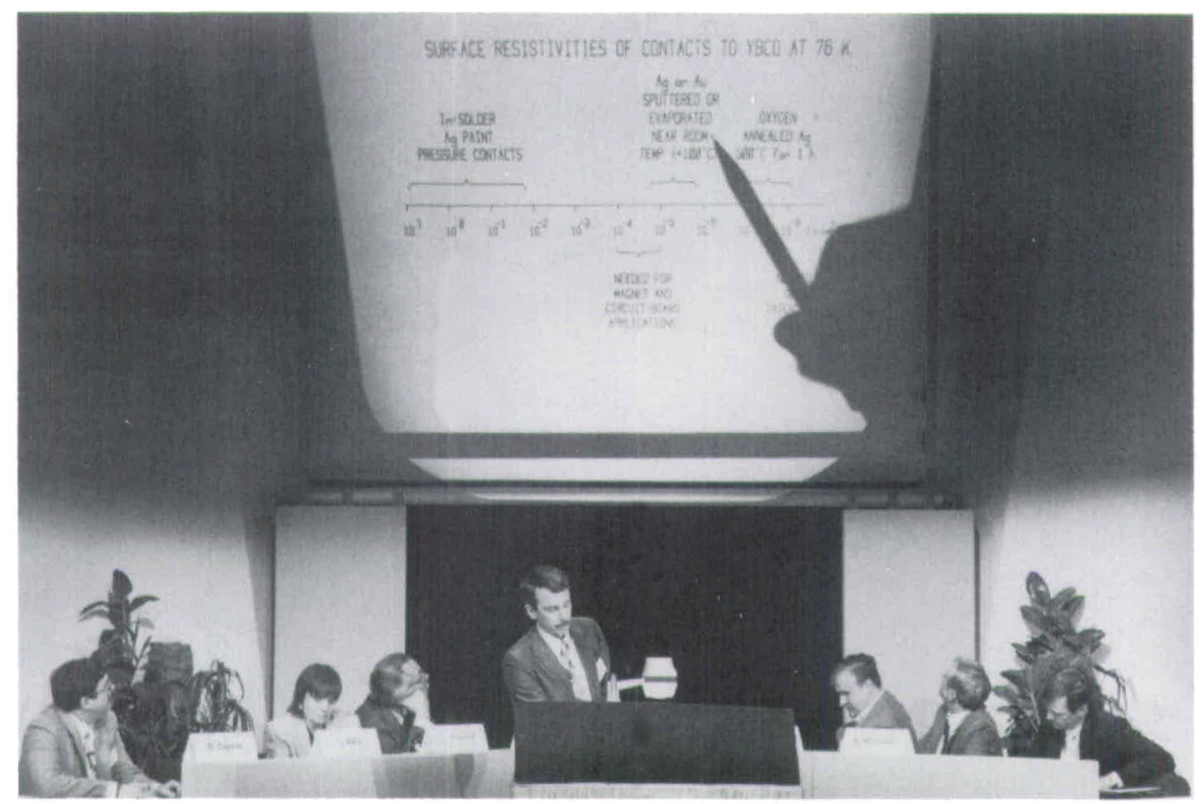

Panel 1 on interconnects and critical currents was beamed via satellite to subscribers across the United States. The speaker is J. Ekin (National Bureau of Standards). Other panelists (from left): D. Capone (Argonne), G. Fisanick (ATET Bell Labs), D. Kroeger (Oak Ridge), panel coordinator J. Kwak (Sandia), R. Landingham (Lawrence Livermore), and W. McCallum (Ames). (Photo, R. Montoya, Sandia)

excess oxygen might be in the form of a superoxide ion.

\section{Panel 3-Oxygen Stoichiometry, Vacancies and Doping}

The third panel considered questions of oxygen stoichiometry, vacancies, and doping. This panel provided some of the most lively discussion of the meeting. D. Morris (Lawrence Berkeley Laboratory) presented work showing a $\Delta \mathrm{T}$ of only a few tenths of a degree for $(>80 \%){ }^{18} \mathrm{O}$ exchanged samples. In contrast, K. Ott (Los Alamos National Laboratory) followed with a discussion of work on the synthesis of $1-2-3$ from ${ }^{17} \mathrm{O}$ and ${ }^{18} \mathrm{O}$ labeled nitrates. The critical temperatures (measured by resistivity) were reduced by tens of degrees for these samples. Unfortunately, time limitations prevented a detailed discussion of this interesting contradiction. Other panelists presented results on the kinetics of oxidation of 1-2-3 using a variety of techniques. According to P. Gallagher (AT\&T Bell Laboratories) an oxidation front is observable in dense samples of 1-2-3. Panelists noted that multiple phases appear when $1-2-3$ is oxygen-loaded to an oxygen stoichiometry greater than 7 .

Panel 4-Pinning, Magnetic Anisotropy and Critical Fields

The fourth panel discussed issues related to pinning, magnetic anisotropy and critical fields. J. Clem (Ames Laboratory) pointed out the granular nature of superconductivity in 1-2-3 materials, with strong superconducting grains and weak coupling, and the need to increase the intergranular current. G. Crabtree (Argonne National Laboratory) demonstrated that flux pinning in single crystals can be increased by neutron irradiation, and G. Venturini (Sandia National Laboratories) presented data for single crystals and ceramics suggesting that different impurity atoms in 1-2-3 can create considerable differences in flux pinning. This illustrates that flux pin- ning can be increased by "finding the right defect." W. Nellis (Lawrence Livermore National Laboratory) suggested that one way of introducing such defects is by dynamic compaction of powders.

\section{Panel 5-Theory}

The final panel discussed issues related to the theory of high temperature superconductors. A. Auerbach (Brookhaven National Laboratory) discussed the low temperature behavior of $2 \mathrm{D}$ antiferromagnets, and $\mathrm{M}$. Weinert (Brookhaven National Laboratory) considered the origin of antiferromagnetic behavior from a band structure point of view. K. Wong (University of Kansas) presented band structure calculations for 1-2-3 and related $\mathrm{CuO}$ and $\mathrm{Cu}_{2} \mathrm{O}$ solids. D. Jennison and E. Stechel (Sandia National Laboratories) described their work on the interactions of the spin subsystem and carriers, suggesting that these interactions introduce spin deviations that lead to pairing. F. Mueller (Los Alamos National Laboratory) commented on the similarity of coherent twin boundaries in orthorhombic 1-2-3 to superlattice structures, suggesting that minigaps due to twins may be present.

The time and place for the next DOE Information Meeting on Superconductivity have not been finalized.

Keith D. Keefer is a member of the technical staff. Ceramics Development Division, Sandia National Laboratories, Albuquerque, NM.

Terry L. Aselage is a member of the technical staff, Electronic Ceramics Division, Sandia National Laboratories, Albuquerque, NM.

\section{Teleconference Enhances Superconductor Info Exchange}

The Fifth DOE Information Meeting on High Temperature Superconductors, January 25-26, 1988 at Sandia National Laboratories was enhanced by a nationwide teleconference. The meeting was televised via the facilities of the Association for Media-based Continuing Education for Engineers, Inc. The TV signal was "uplinked" to satellite G-Star I in geosynchronous orbit 22,300 miles above the earth, and the broadcast was beamed to industry and university members of the National Technical University. Two telephones at Sandia were dedicated to questions for the conference panelists from teleconference participants.

Based on the most recent figures, 235 people in 26 organizations participated in the conference via satellite. Some of the universities that picked up the teleconference were Purdue, Auburn, Iowa State, Oklahoma State, and Southern Methodist. Industry participants included Digital, Polaroid, GE, duPont, Allied Signal, and Hewlett-Packard. Two national laboratories, Lawrence Berkeley and Los Alamos, also participated. (Taken from Sandia Lab Nezus, Vol. 40, No. 3.) 



\section{Megavolt ion implantation}

There are various examples in which MeV ion implantation offers the opportunity for unique device structures and material modifications. These include among others direct formation of buried collectors and barrier layers, production of isolation wells in CMOS devices, the programming of ROM transistors and modification of metals, ceramics and polymers.

Because the use of multiply charged ions has several drawbacks such as low beam current, charge exchange problems and the inefficient use of source output, High Voltage Engineering Europa B.V. has developed single ended $1 \mathrm{MV}$ and $2 \mathrm{MV}$ accelerators for ion implantation. Compared with other systems this design has the advantage of covering a range of $30-1000 \mathrm{kV}$ or $50-2000 \mathrm{kV}$, which span exactly the gap between energies which are often required.

As the energy stability of both systems is better than $\pm 1 \mathrm{kV}$ at maximum voltage level, they are also fully adequate for ion beam analysis including Rutherford Backscattering Spectroscopy (RBS), Particle Induced X-ray Emission (PIXE) and Nuclear Reaction Analysis (NRA).

Single ended accelerator

- Wide energy range

- Simple ion optics

- Ion source exchange system

- Compatible with four types of ion sources

- Pre-analyzing Wien filter

- No conditioning and virtually no shielding

System for ion implantation

- Ultra clean (class 10) operation

- Cassette to cassette/Return within a cassette operation

- Implantation angle and flat angle per wafer/batch programmable

- Si and GaAs wafers up to incl. $150 \mathrm{~mm}$

- GCA or Fluoroware type $\mathrm{H}$ bar cassettes

- Wafer throughput not limited by elevator pumping time 